\title{
Biocompatible PEG-Chitosan@Carbon Dots Hybrid Nanogels for Two-Photon Fluorescence Imaging, Near-Infrared Light/pH Dual-Responsive Drug Carrier, and Synergistic Therapy
}

\author{
Hui Wang, Jing Di, Yubing Sun, Jianping Fu, Zengyan Wei, Hiroshi Matsui, \\ Alejandra del C. Alonso, and Shuiqin Zhou*
}

This work designs a class of biocompatible PEG-chitosan@CDs hybrid nanogels by integrating nonlinear poly(ethylene glycol) (PEG), chitosan, and graphitic carbon dots (CDs) into a single nanoparticle for two-photon fluorescence (TPF) bioimaging, $\mathrm{pH}$ and near-infrared (NIR) light dual-responsive drug release, and synergistic therapy. Such hybrid nanogels can be simply prepared from a one-pot surfactant-free precipitation polymerization of the PEG macromonomers complexed with chitosan and CDs in water, resulting in a semi-interpenetration of chitosan chains and an immobilization of CDs in the nonlinear PEG networks. The embedded CDs in hybrid nanogels not only serve as an excellent confocal and TPF imaging contrast agent and fluorescent $\mathrm{pH}$-sensing probe, but also enhance the loading capacity of the hybrid nanogels for hydrophobic anticancer drug. The chitosan can induce a $\mathrm{pH}$-sensitive swelling/deswelling of the hybrid nanogels for $\mathrm{pH}$-regulated drug release over the physiologically important range of 5.0-7.4 and surface modulation of embedded $\mathrm{CD}$ s to realize fluorescent $\mathrm{pH}$ sensing. The thermosensitive nonlinear PEG network can promote the drug release through the local heat produced by the embedded CDs under NIR irradiation. The in vitro results indicate that the hybrid nanogels demonstrated high therapeutic efficacy through the synergistic effect of combined chemo-photothermal treatments.

\section{Introduction}

Stimuli-responsive nanogels have gained significant progress because of their great potential for applications in intelligent drug delivery and other biomedical fields. ${ }^{[1-4]}$ Recently, much attention has been focused on the integration of stimuli-responsive polymer nanogels with inorganic nanoparticles (NPs) to combine the biosensing or bioimaging ability with the controlled drug delivery function. ${ }^{[5-10]}$ A key attribute of such responsive hybrid nanogels as drug delivery systems is their ability to regulate the drug release under specific environments or stimuli, which can significantly improve the therapeutic efficacy at pathological sites but reduce the systemic side effects. ${ }^{[11]}$ Both endogenous and exogenous activations can be used to regulate the drug release from their carriers. ${ }^{[12-14]}$ The endogenous activation can be realized by the variation of specific physiochemical characteristics of the pathological microenvironment. ${ }^{[15-17]}$ For example, many pathological processes in tumor tissue and intracellular endosome/lysosome decrease the local $\mathrm{pH}$ by $1-2.5 \mathrm{pH}$ units or increase the local temperature by $1-5{ }^{\circ} \mathrm{C}$ in comparison with that of blood and normal tissues. ${ }^{[18-20]}$ On the other hand, the exogenous activation that can remotely control the drug release at a desired site and time is considered crucial to boost the drug efficacy in cancer treatment while minimizing side effects. ${ }^{[21-26]}$ Among all external stimuli including light and magnetic field, near-infrared (NIR) light is the most favored for controlling the drug release due to its simple operation, low energy absorption, maximum penetration, and minimum side effects for human tissue and organs. ${ }^{[27,28]}$ So far, nanostructured gold $(\mathrm{Au})$ has been commonly integrated into the stimuli-responsive polymer nanogels to realize simultaneous NIR light-triggered drug release and cell imaging. ${ }^{[2-34]}$ However, several disadvantages are associated with the noble metal NPs for their use in biomedical areas, including the 
toxicity for neuronal cells, potential induction of oxidative stress processes in the body, and high cost. ${ }^{[35,36]}$

Recently, small carbon dots (CDs) have received tremendous attention for their unique combination of excellent photostability, small size, biocompatibility, highly tunable photoluminescence (PL) property, and chemical inertness. ${ }^{[37-48]}$ Compared to noble metal NPs, CDs not only demonstrate lower toxicity and better biocompatibility according to their cytotoxic and in vivo toxic evaluation results but also possess many hydrophilic surface functional groups and thus can be easily dispersed in aqueous media without the need for further surface modification. With these superior properties combined in a single dot, CDs have been recently evaluated as bioimaging agents. ${ }^{[41-47]}$ In addition, CDs can be used for photothermal therapy and two-photon fluorescent (TPF) imaging as a result of their absorption for NIR light and upconversion PL properties. ${ }^{[49-53]}$ Considering the unique combination of these key material properties of CDs, it is desirable to integrate the CDs with biocompatible stimuli-responsive nanogels to develop a new type of multifunctional nanoplatform. It is expected that such integrated hybrid nanogels can not only serve as an optical code for two-photon bioimaging and biosensing but also realize both endogenous and exogenous triggered drug release to enhance the therapeutic efficacy.

Herein, we design a new class of biocompatible hybrid nanogels by immobilizing the fluorescent CDs into biocompatible temperature/pH dual-responsive polymer nanogels for simultaneous fluorescent $\mathrm{pH}$ sensing, TPF imaging, NIR light, and $\mathrm{pH}$ dual-responsive drug delivery. With a rational design on the compositions and properties of each building block, such a hybrid nanogel can be synthesized using a simple one-pot surfactant-free precipitation polymerization in water. Specifically, poly(ethylene glycol) (PEG) macromonomers and crosslinkers, chitosan chains, and CDs bearing surface hydroxyl/carboxyl groups were all dissolved in water. The hydroxyl/amine groups on the repeating units of chitosan chains, the surface hydroxyl/ carboxyl groups on CDs, and the ether oxygen on the PEG macromonomers can form hydrogen bonding associations or complexation. When these PEG macromonomers are polymerized and crosslinked, the chitosan chains will be physically entangled and interpenetrated in the resultant nonlinear PEG chain networks and the CDs will be in situ immobilized in the polymerized gel networks. These designed building blocks have not only excellent biocompatibility but also unique properties and functions. As schematically depicted in Figure 1, the newly designed hybrid nanogels combining the functional building blocks of fluorescent CDs, thermoresponsive nonlinear PEG,

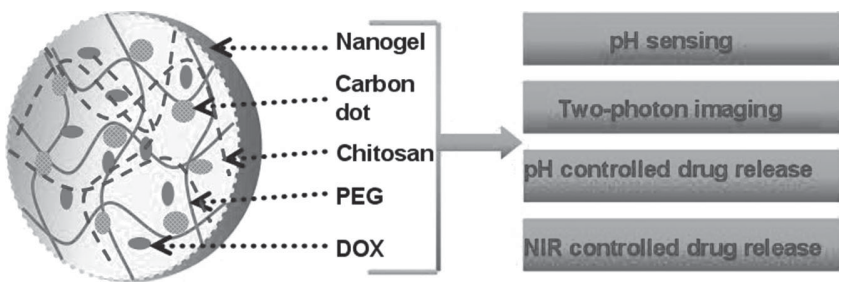

Figure 1. Schematic illustration of biocompatible PEG-chitosan@CDs hybrid nanogel for multifunctional applications in biomedical field, with CD representing for carbon dot. and $\mathrm{pH}$-responsive chitosan should provide great potential for biomedical applications. We demonstrate that the tri-component hybrid nanogels, denoted as PEG-chitosan@CDs, display cooperative properties while maintaining the individual properties of each constituent, thus allowing the integration of several important functions. First, the chitosan-penetrated hybrid nanogels can not only sense the changes in environmental $\mathrm{pH}$ over the physiologically important range of 5.0-7.4 but also enable a biologically regulated release of the loaded anticancer drug Doxorubicin (DOX) due to the specific disruptions in the acid/base homeostasis of the local pathological environment (endogenous activation). Second, the hybrid nanogels composed of thermoresponsive nonlinear PEG network can trigger the drug release under the localized hyperthermia induced by an external irradiation of NIR light benefited from the photothermal conversion ability of the embedded CDs (exogenous activation). In addition, the biocompatible hybrid nanogels can overcome cellular barriers to enter the intracellular region, which not only serve as a regular fluorescent cellular imaging agent under an excitation wavelength of $405 \mathrm{~nm}$ but also serve as a TPF imaging agent under an excitation wavelength of NIR light (900 nm). Such a multifunctional intelligent hybrid nanogel demonstrates a great promise toward the advanced nanoplatform for simultaneous medical diagnosis and high efficacy therapy.

\section{Results and Discussion}

The biocompatible PEG-chitosan@CDs hybrid nanogels are synthesized by one-pot surfactant-free precipitation polymerization and crosslinking of the PEG comonomers [(2-(2-methoxyethoxy)ethyl methacrylate $\left(\mathrm{MEO}_{2} \mathrm{MA}\right)$ and oligo(ethylene glycol) methyl ether methacrylate $\left.\left(\mathrm{MEO}_{5} \mathrm{MA}\right)\right]$ complexed with chitosan chains and $\mathrm{CDs}$ in water $(\mathrm{pH} \approx 5.8)$ at $70.0{ }^{\circ} \mathrm{C}$. While chitosan chains carry hydroxyl $(-\mathrm{OH})$ and amine $\left(-\mathrm{NH}_{2}\right)$ groups in the repeating units, the CDs synthesized from the acid-assisted hydrothermal decomposition of glucose possess hydrophilic surface $-\mathrm{OH} /-\mathrm{COOH}$ groups (see Fourier transform infrared, FTIR, spectrum of CDs at 3229 and $1708 \mathrm{~cm}^{-1}$ in Figure S2 in the Supporting Information). It is expected that both the chitosan chains and CDs will complex with the $\mathrm{MEO}_{2} \mathrm{MA}$ and $\mathrm{MEO}_{5} \mathrm{MA}$ comonomers through the hydrogen bonding associations among the $-\mathrm{OH} /-\mathrm{NH}_{2}$ groups of chitosan, $-\mathrm{OH} /-\mathrm{COOH}$ groups of $\mathrm{CDs}$, and the ether oxygens of the $\mathrm{MEO}_{2} \mathrm{MA}$ and $\mathrm{MEO}_{5} \mathrm{MA}$ monomers. ${ }^{[54]}$ When these PEG macromonomers are polymerized and crosslinked, the complexed chitosan chains and CDs will be in situ trapped in the resulted nonlinear PEG networks. The chitosan $\left(\mathrm{p} K_{\mathrm{a}} \approx 6.2\right)$ with small degree ionization of the amine groups at the reaction medium can stabilize the resultant PEG-chitosan@CDs hybrid nanogel particles, thus no surfactant was needed for the synthesis in water. A comparison of the FTIR spectra of the free CDs, PEG-chitosan nanogels, and PEG-chitosan@CDs hybrid nanogels (Figure S2, Supporting Information) indicates that the hybrid nanogels exhibit the characteristic peaks from both free CDs and PEG-chitosan nanogels. Figure 2A compares the typical UV-vis absorption spectra of the CDs, PEG-chitosan nanogels, and PEG-chitosan@CDs hybrid nanogels. While the PEG-chitosan nanogels have no significant absorption at 

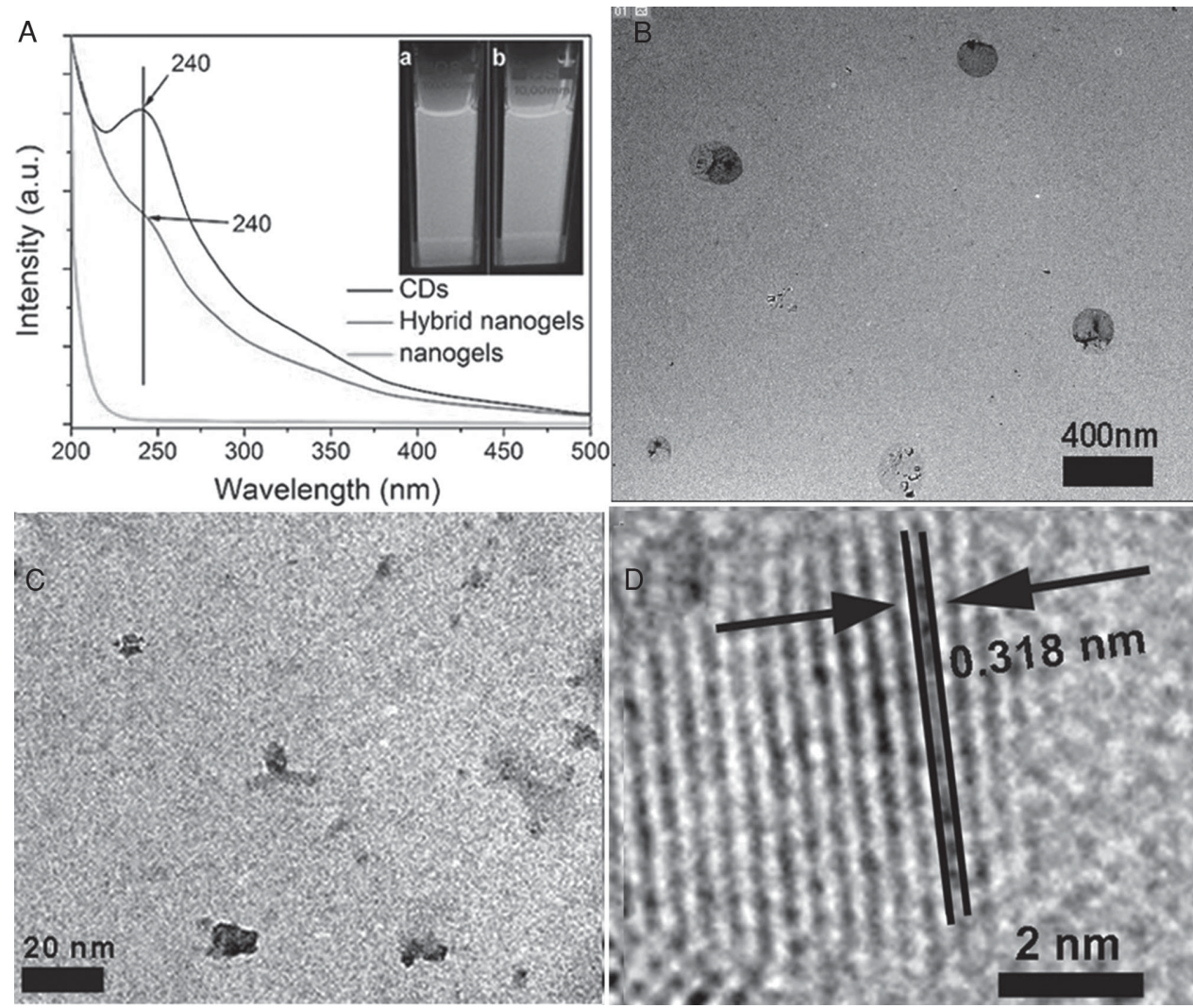

Figure 2. A) UV-Vis spectra of the CDs, PEG-chitosan nanogels, and PEG-chitosan@CDs hybrid nanogels. The insets a and b show photographs of the aqueous dispersions of the purified hybrid nanogels under a UV light (365 nm) before and after 30 d dialysis; B) TEM image of the PEG-chitosan@ CDs hybrid nanogels; C) highly magnified sectional TEM image of a typical PEG-chitosan@CDs hybrid nanogel; D) lattice fringe of a single CD under high resolution TEM.

wavelength above $240 \mathrm{~nm}$, the free CDs exhibit a clear broad peak centered at $240 \mathrm{~nm}$, which represents the typical absorption of an aromatic Pi system and is similar to that of polycyclic aromatic hydrocarbons. ${ }^{[55]}$ Compared to the free CDs and PEGchitosan nanogels, the synthesized hybrid nanogels show a similar absorption peak centered at $240 \mathrm{~nm}$, indicating that the CDs have been successfully immobilized into the PEG-chitosan networks. When the aqueous dispersion of these hybrid nanogels was exposed to UV light (365 nm), green-blue light was obviously emitted (inset "a" in Figure 2A). It should be mentioned that such designed PEG-chitosan@CDs hybrid nanogels have very stable optical property because of the in situ immobilization of the CDs in the PEG-chitosan polymer network via hydrogen bonding interactions. No obvious fluorescence intensity change was observed after the hybrid nanogels were continuously dialyzed for $30 \mathrm{~d}$ against frequently changed water and (inset "b" in Figure 2A). Figure 2B shows a typical TEM image of the as-synthesized PEG-chitosan@CDs hybrid nanogels at dried state. The hybrid nanogels display a quasispherical morphology with an average diameter of about $182 \mathrm{~nm}$ and a remarkably heterogeneous structure with randomly dispersed dark spots/domains distributed in a relatively light-contrasted gray matrix. The dark spots/domains might be attributed to the individual and associated CDs embedded in the interior of polymer networks. The highly magnified sectional TEM image of a typical PEG-chitosan@CDs hybrid nanogel particle
(Figure 2C) confirms that many black dots with size in a range of $3-10 \mathrm{~nm}$ are randomly distributed in the polymer matrix. The small black dots under high-resolution TEM exhibit a nanocrystal structure with 2D lattice fringes showing the interplanar distance of about $0.318 \mathrm{~nm}$ (Figure 2D), which corresponds to the (002) lattice planes of graphitic $\left(\mathrm{sp}^{2}\right)$ carbon. ${ }^{[56]}$ These TEM results further confirm that the graphitic CDs have been successfully immobilized into the PEG-chitosan networks.

We expect that the PEG-chitosan@CDs hybrid nanogels containing the ionizable $-\mathrm{NH}_{2}$ groups from the interpenetrated chitosan chains should exhibit $\mathrm{pH}$-responsive swelling/ deswelling transitions. Figure $3 \mathrm{~A}$ shows the average hydrodynamic diameter $\left(D_{\mathrm{h}}\right)$ value of the resultant PEG-chitosan@ CDs hybrid nanogels dispersed in solutions of different $\mathrm{pH}$ values, measured at a scattering angle of $\theta=30^{\circ}$ and the physiological temperature of $37.0^{\circ} \mathrm{C}$. Clearly, the increase in the $\mathrm{pH}$ value of dispersion medium can gradually shrink the size of the PEG-chitosan@CDs hybrid nanogels with a dramatic size decrease occurring in the $\mathrm{pH}$ range of 5.0-6.2. It is understandable to observe this critical volume transition $\mathrm{pH}$ range for the PEG-chitosan@CDs hybrid nanogels. At pH below the $\mathrm{p} K_{\mathrm{a}}$ of chitosan $(\approx 6.2)$, the $-\mathrm{NH}_{2}$ groups on the chitosan chains are protonated and positively charged, which produce strong Coulomb forces. The mobile counterions cannot leave this phase to preserve the charge neutrality of the gel, therefore, produce full osmotic pressure for the gel to swell. ${ }^{[57]}$ At $\mathrm{pH}$ below 5.0, 

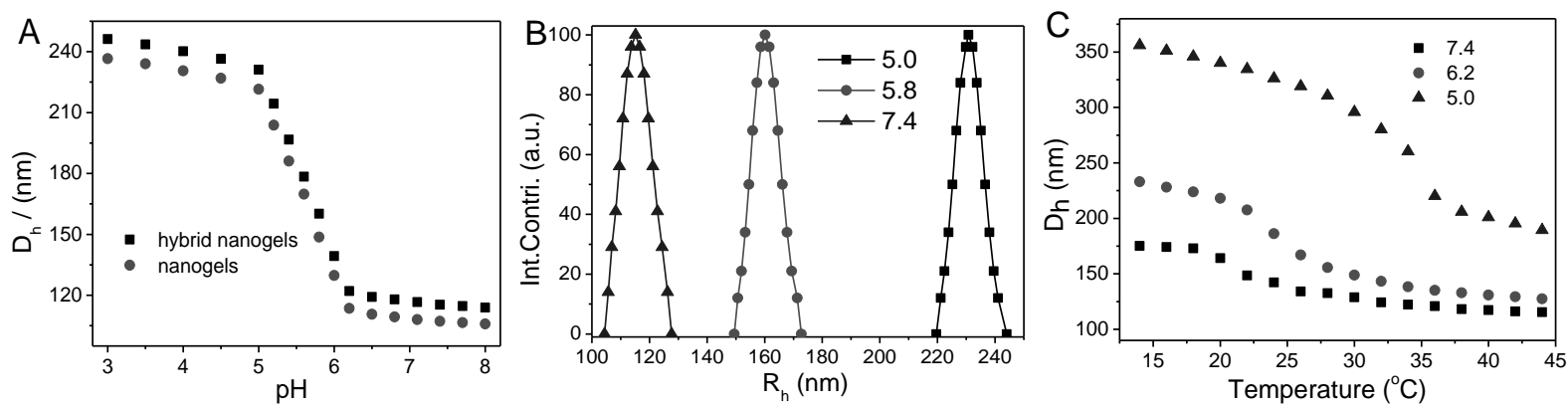

Figure 3. A) $\mathrm{pH}$-induced size $\left(D_{h}\right)$ change of PEG-chitosan nanogels and PEG-chitosan@CDs hybrid nanogels at $37.0^{\circ} \mathrm{C}$; $\left.\mathrm{B}\right)$ size distributions of PEG-chitosan@CDs hybrid nanogels at $\mathrm{pH}=5.0,5.8$, and 7.4, respectively; $\mathrm{C}$ ) temperature-induced size change of the hybrid nanogels at different $\mathrm{pH}$ values. All measurements were made at a scattering angle $\theta=30^{\circ}$.

the $-\mathrm{NH}_{2}$ groups on the chitosan chains are nearly fully protonated, thus the hybrid nanogels swell to nearly a maximum degree. Based on this swelling mechanism, the PEG-chitosan nanogels prepared under the same conditions but without the addition of CDs exhibit a similar pH-dependent swelling curve to that of the PEG-chitosan@CDs hybrid nanogels. However, we found that the PEG-chitosan@CDs hybrid nanogels display larger sizes than the PEG-chitosan nanogels under all the measured $\mathrm{pH}$ values due to the successful embedding of the CDs in the PEG-chitosan@CDs hybrid nanogels. It should be mentioned that the resultant hybrid nanogels demonstrate a very narrow size distribution regardless of their swelling or shrinking states under different $\mathrm{pH}$ value, as shown in Figure 3B. In addition, the surrounding temperature can further manipulate the size of the PEG-chitosan@CDs hybrid nanogels (Figure 3C). It is known that the nonlinear PEG chains can undergo a thermoresponsive volume phase transition as a consequence of the counterbalance of hydrophilic (PEG side chains) and hydrophobic (carbon backbone) forces in water. ${ }^{[58]}$ For the PEG-chitosan@CDs hybrid nanogels, the critical volume phase transition temperature also depends on the $\mathrm{pH}$-dependent ionization degree of the gel. The higher the ionization degree of the chitosan chains (e.g., pH below 5.0), the more hydrophilic of the hybrid nanogels, which requires a higher critical volume phase transition temperature for the nonlinear PEG networks chains to shrink. ${ }^{[59]}$ At the body temperature of $37.0{ }^{\circ} \mathrm{C}$, the hybrid nanogels displayed an overall smaller swelling ratio at the physiological $\mathrm{pH}=7.4$ than at $\mathrm{pH}=$ 5.0. These results indicate that the hydrogen-bonding association and entanglement interactions not only enable the hybrid nanogels to exhibit $\mathrm{pH} /$ thermo dual-responsive phase behavior but also allow us to effectively modulate the $\mathrm{pH}$ response of the hybrid nanogels by applying cooling/heating strategy.

With the fluorescent CDs successfully embedded into the PEG-chitosan networks, it is expected that the PEG-chitosan@ CDs hybrid nanogels will demonstrate the similar tunable and upconverted PL properties to the free CDs. Figure 4A shows the typical PL spectra of the PEG-chitosan@CDs hybrid nanogels under different excitation wavelengths $\left(\lambda_{\text {ex }}\right)$ in the UV-vis wavelength range. When the $\lambda_{\text {ex }}$ increases from 260 to $520 \mathrm{~nm}$, the emissions redshifted to longer wavelengths, which corresponds to the different surface energy traps of the CDs. ${ }^{[60]}$ The multiwavelength excitation spectra of the PEG-chitosan@CDs hybrid nanogels (Figure S3, Supporting Information) confirm the different surface energy traps of these CDs. The maximum PL emission intensity locates at $477 \mathrm{~nm}$, which was obtained with $\lambda_{\text {ex }}=400 \mathrm{~nm}$. Figure 4B shows the PL spectra of the PEGchitosan@CDs hybrid nanogels under the excitations in the visNIR wavelength range. The upconverted emissions located in the range from 535 to $443 \mathrm{~nm}$ were clearly demonstrated when the $\lambda_{\text {ex }}$ was applied from 980 to $620 \mathrm{~nm}$, which should be attributed to the multiphoton active process similar to previously reported CDs. ${ }^{[61]}$ In addition, the PEG-chitosan@CDs hybrid nanogels also exhibit excellent photostability. The PL spectra of the hybrid nanogels obtained under a continuous excitation of UV light $\left(\lambda_{\mathrm{ex}}=360 \mathrm{~nm}\right)$ for $2 \mathrm{~h}$ (Figure S4A) change slightly. The maximum PL intensity obtained at emission wavelength $\left(\lambda_{\text {em }}\right)$ of $450 \mathrm{~nm}$ decreases by $\approx 3.7 \%$ after $2 \mathrm{~h}$ continuous exposure to the excitation light of $360 \mathrm{~nm}$ (Figure S4B, Supporting Information). All these results indicate that the as-obtained PEG-chitosan@CDs hybrid nanogels not only demonstrate $\lambda_{\text {ex }}$-tunable and upconverted PL properties but also show excellent photostability against light illumination and air exposure, which is necessary for bioimaging and biosensing applications. Figure 4C depicts the $\mathrm{pH}$-dependent PL spectra of the hybrid nanogels obtained with $\lambda_{\mathrm{ex}}=360 \mathrm{~nm}$. It is clear that the increase in $\mathrm{pH}$ from 2.99 to 8.00 induces a significant decrease in the PL intensity of the PEG-chitosan@CDs hybrid nanogels. Meanwhile, the emission peak also slightly redshifts from 438 to $444 \mathrm{~nm}$. As we previously discussed for Figure 3A, the $\mathrm{pH}$ change of the dispersion medium can induce a volume phase transition of the hybrid nanogels. To visualize how the $\mathrm{pH}$ induced PL intensity change is correlated to the $\mathrm{pH}$-induced volume phase transitions of the hybrid nanogels, we plotted the relative maximum $\mathrm{PL}$ intensity as a function of $\mathrm{pH}$ (Figure 4D). Interestingly, the comparison of Figure 4D with Figure 3A indicates that a conspicuous decrease in the PL intensity of the hybrid nanogels occurred at nearly the same $\mathrm{pH}$ range (5.0-6.2) as that of the gradual decrease in the size of the hybrid nanogels. This result implies that the PL quenching of the embedded $\mathrm{CDs}$ in the hybrid nanogels is caused by the $\mathrm{pH}$-induced deionization shrinking of the PEG-chitosan nanogels. It has been reported that the PL of CDs is more of a surface process as a radiative recombination of the surface-confined electrons and holes instead of depending on $\mathrm{sp}^{2}$ clusters in the core. ${ }^{[42,45]}$ For example, the surface passivation with PEG polymer can 

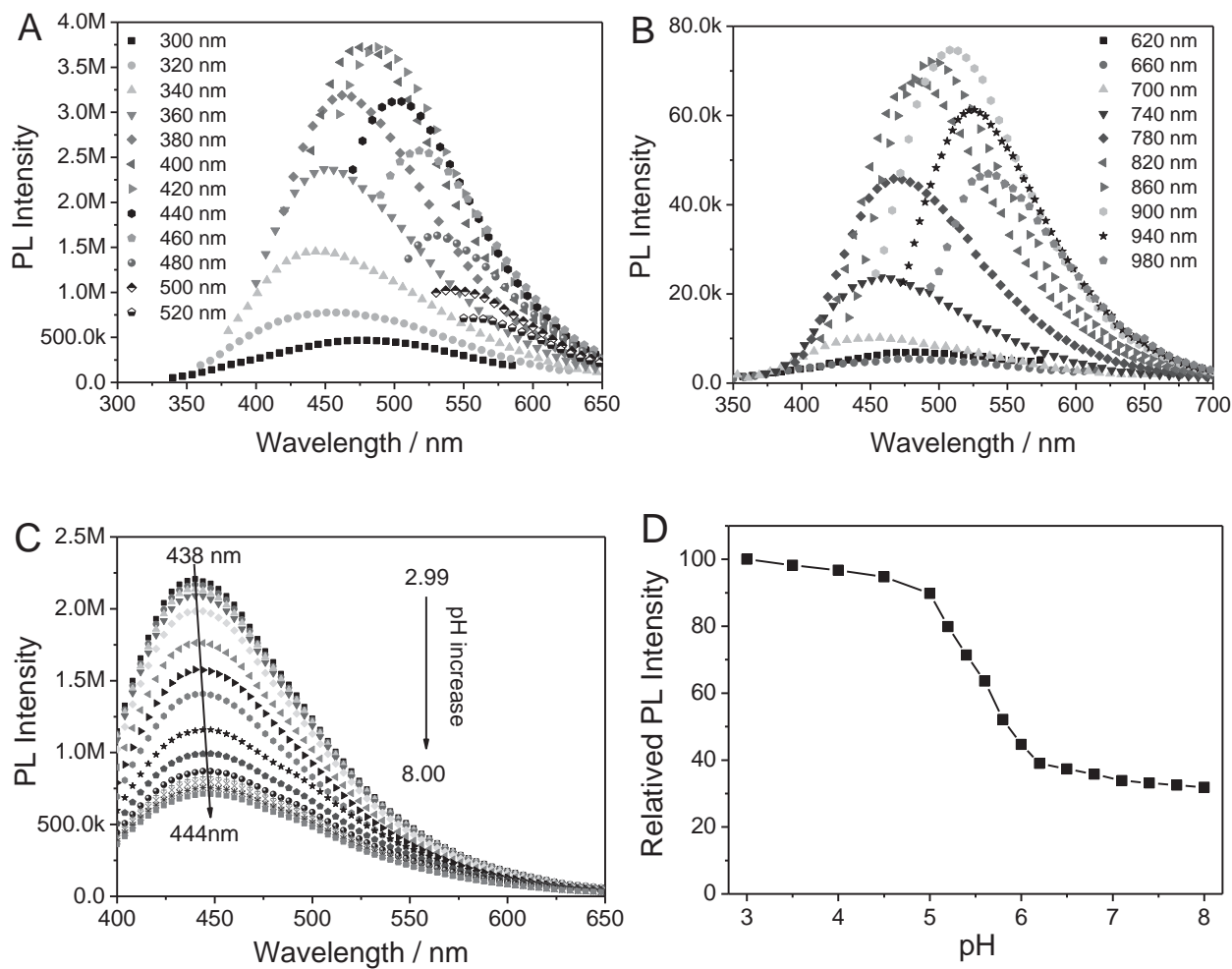

Figure 4. A,B) PL spectra and upconverted PL spectra of the PEG-chitosan@CDs hybrid nanogels under different excitation wavelengths $\left(\lambda_{\mathrm{ex}}\right)$ in the UV-vis and vis-NIR ranges, respectively; C,D) PL spectra and relative maximum PL intensity of the PEG-chitosan@CDs hybrid nanogels obtained at $\lambda_{\text {ex }}=360 \mathrm{~nm}$ under different $\mathrm{pH}$ values.

dramatically enhance the PL intensity of CDs. ${ }^{[62]}$ For the samesized CDs, the overall PL properties of CDs are determined by the competition between the emissive sites and nonradiative trap sites on the surface. ${ }^{[47]}$ In our PEG-chitosan@CDs hybrid nanogels, the CDs are immobilized in the polymer network via secondary force between their surface $-\mathrm{OH} /-\mathrm{COOH}$ groups and the $-\mathrm{OH} /-\mathrm{NH}_{2}$ groups of chitosan chains as well as the ether oxygen of nonlinear PEG chains. At pH below 5.0, the fully protonated $-\mathrm{NH}_{3}{ }^{+}$groups of the chitosan chains have strong electrostatic attractions with the dissociated surface $-\mathrm{COO}^{-}$ groups on CDs, thus surface of CDs is well passivated by the polymer chains. When the $\mathrm{pH}$ was increased from 5.0 to 6.2 , the $-\mathrm{NH}_{3}{ }^{+}$groups of chitosan gradually change to neutral $-\mathrm{NH}_{2}$ groups, which reduce the electrostatic attractions between chitosan chains and CDs. In other words, the chitosan chains are less attached on the surface of CDs when $\mathrm{pH}$ changes from 5.0 to 6.2. The less passivated CDs may have more nonradiative trap sites and thus resulting in the quenched PL intensity. Since the $\mathrm{pH}$-induced protonation/deprotonation of the $-\mathrm{NH}_{2}$ groups on chitosan chains is reversible, we expect that the quenched PL intensity should be recovered by changing the $\mathrm{pH}$ back to below 5.0 , at which the positively charged chitosan chains can passivate the surface of CDs well. To examine the reversibility of the pH-induced PL change, the PL spectra of the PEG-chitosan@ CDs hybrid nanogels were measured for six cycles with a $\mathrm{pH}$ adjustment between 2.99 and 8.00 through centrifugation and redispersion in buffer solutions. As shown in Figure S5 (Supporting Information), the maximum PL intensity of the hybrid nanogels is fully reproducible after the repeated acidification and alkalization of the dispersion solutions, which indicates that the CDs have been stably immobilized in the PEG-chitosan chain networks. The reversible optical property change is critical for the hybrid nanogels to serve as a sensor.

After confirming the strong fluorescence and tunable emissions of the PEG-chitosan@CDs hybrid nanogels, DU145 human prostate cancer cells were selected as a model to evaluate the optical cellular imaging label function of these hybrid nanogels. Figure 5 shows the laser scanning confocal images of the DU145 cells incubated with the PEG-chitosan@CDs hybrid nanogels under an excitation wavelength of $405 \mathrm{~nm}$ for different irradiation time. The PEG-chitosan@CDs hybrid nanogels emit a bright fluorescence and can light up the DU145 cells. In addition, the confocal images of DU145 cells did not show fluorescent signal change after a continuous irradiation with the excitation laser at $405 \mathrm{~nm}$ for $30 \mathrm{~min}$, which further indicates that the PEG-chitosan@CDs hybrid nanogels have excellent photostability and can be used for long-term cellular imaging.

Given the obviously observed upconverted PL properties in Figure 4B, the TPF cell imaging ability of the PEG-chitosan@ CDs hybrid nanogels was expected. Figure 6 shows the transmission (A), TPF (B), and their overlaid (C) images of the DU145 human prostate cancer cells after uptaking the PEGchitosan@CDs hybrid nanogels upon an excitation by a femtosecond infrared laser of $900 \mathrm{~nm}$. It is clear that the cells can be illuminated by the upconverted fluorescence emitted from the hybrid nanogels under the excitation of an NIR laser. In addition, the 3D cell imaging video (Figure S6, Supporting 


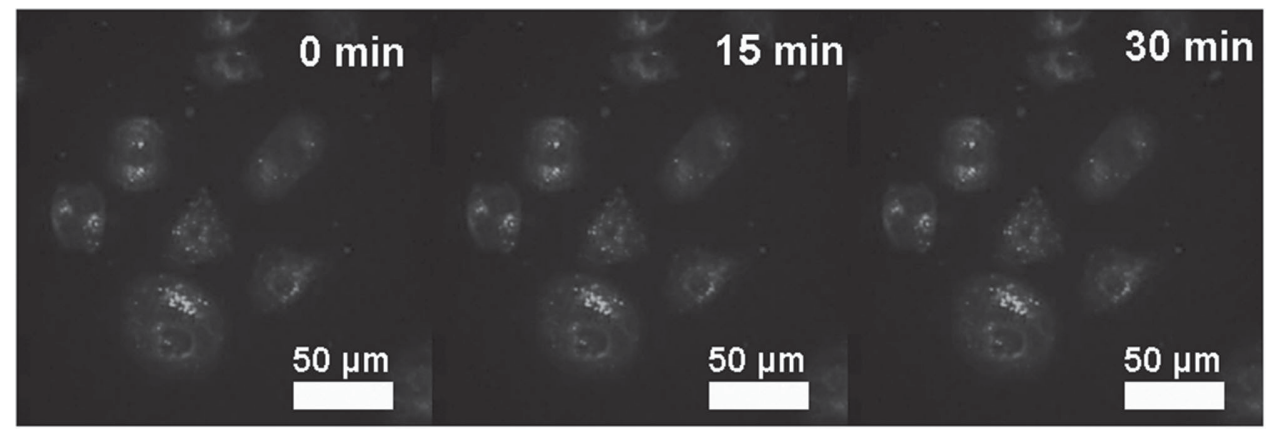

Figure 5. Laser scanning confocal microscopy images of DU145 human prostate cancer cells incubated with the PEG-chitosan@CDs hybrid nanogels $\left(5 \mu \mathrm{g} \mathrm{mL} \mathrm{L}^{-1}\right)$ under an excitation wavelength of $405 \mathrm{~nm}$ for different exposure time.

Information) of the DU145 human prostate cancer cells incubated with the hybrid nanogels demonstrates that the hybrid nanogels indeed enter into the intracellular region and have a high signal-to-noise ratio. The hybrid nanogels are distributed in the cytoplasm but not in the karyons. As the complexity of molecular interactions governing endocytosis is revealed, the mechanisms of endocytosis of the hybrid nanogels should be viewed in a broader context than simple vesicular trafficking. ${ }^{[63,64]}$ All these results indicate that the PEG-chitosan@ CDs hybrid nanogels could be potentially used as a transmembrane drug carrier to release the drug molecules inside the tumor cells and realize a chemotherapy. The novel fluorescent property of the PEG-chitosan@CDs hybrid nanogels offers a multiple-choice platform for biological labels.

The demonstrated $\mathrm{pH}$-responsive volume phase transition and the endocytosis ability imply that the PEG-chitosan@CDs hybrid nanogels could be a promising smart drug carrier. Here, DOX is selected as an anticancer drug to test the drug-loading capacity and release behavior of the PEG-chitosan@CDs hybrid nanogels. Results show that the DOX molecules can be readily loaded into the PEG-chitosan@CDs hybrid nanogels by a simple mixing of the hybrid nanogels into the PBS solution of DOX. The DOX-loaded hybrid nanogels clearly demonstrate a UVvis absorption peak at around $480 \mathrm{~nm}$ from the characteristic absorption of DOX molecules (Figure S7, Supporting Information). The quantitative analysis determines that the loading content of DOX in the PEG-chitosan@CDs hybrid nanogels and PEG-chitosan nanogels are 33 and $21 \mathrm{mg} \mathrm{g}^{-1}$, respectively. This result indicates that the CD-containing hybrid nanogels have a much higher loading capacity for DOX drug molecules than the CD-free PEG-chitosan nanogels. This increased DOX loading capacity of the PEG-chitosan@CDs hybrid nanogels should be attributed to the conjugated aromatic carbon structures of the CDs embedded in the gel network, which can provide supramolecular $\pi$ stacking interactions with the DOX molecules and thus entrap more DOX molecules inside the polymer gel network. ${ }^{[65]}$

Figure 7A shows the $\mathrm{pH}$-responsive DOX releasing profiles from the PEG-chitosan@CDs hybrid nanogels obtained at different $\mathrm{pH}$ values of 5.0,6.2, and 7.4, respectively. At the physiological pH of 7.4 , only $20.8 \%$ of loaded DOX was released from the hybrid nanogels at $37{ }^{\circ} \mathrm{C}$ after $96 \mathrm{~h}$. With the $\mathrm{pH}$ decreased to 6.2 , the amount of released DOX was increased to $25.4 \%$ after $96 \mathrm{~h}$. In contrast, the drug releasing rate at $\mathrm{pH}=5.0$ dramatically increased. About $49.2 \%$ of the loaded DOX molecules could be released from the hybrid nanogels after $96 \mathrm{~h}$, which indicates that the drug release rate can be easily regulated by varying the $\mathrm{pH}$ value of the releasing medium. The observed $\mathrm{pH}$ dependency of the DOX releasing rate should be associated with a few factors. First, the decrease in $\mathrm{pH}$ from 6.2 to 5.0 induces the protonation of the $-\mathrm{NH}_{2}$ groups of chitosan chains and thus a swelling of the PEG-chitosan@CDs hybrid nanogels, which can provide more paths for the loaded DOX molecules to diffuse out from the gel network. Second, DOX is a weak amphipathic base with $\mathrm{a} \mathrm{p} K_{\mathrm{a}} \approx 8.2$. The decrease in $\mathrm{pH}$ from 6.2 to 5.0 enables a more completed protonation of the $-\mathrm{NH}_{2}$ group on DOX molecules, which not only significantly increases the solubility/ hydrophilicity of the DOX molecules but also increases the electrostatic repulsions between the DOX molecules and the

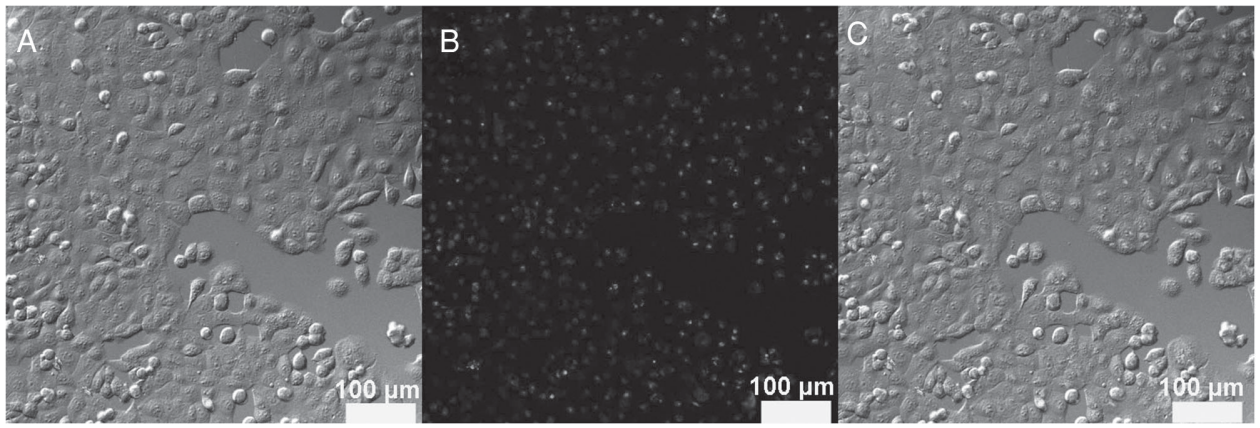

Figure 6. A) Transmission, B) two-photon fluorescence, and C) overlaid images of DU145 human prostate cancer cells incubated with PEG-chitosan@ CDs hybrid nanogels. Excitation laser wavelength is $900 \mathrm{~nm}$. 

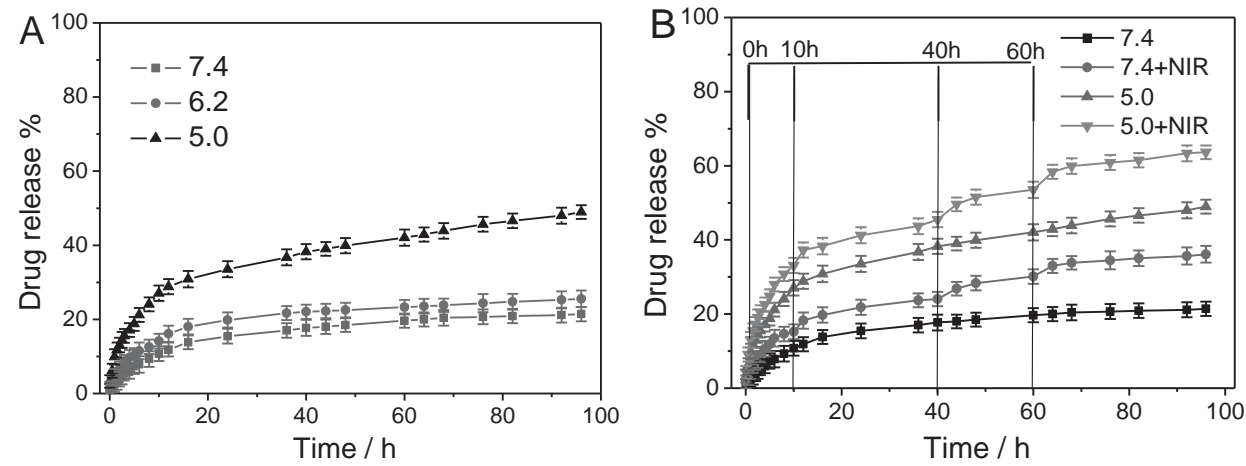

Figure 7. A) The $\mathrm{pH}$-dependent release of DOX from the PEG-chitosan@CDs hybrid nanogels under different $\mathrm{pH}$ values $(7.4,6.2$, or 5$)$ at $37{ }^{\circ} \mathrm{C}$; B) releasing profiles of DOX from the hybrid nanogels in $\mathrm{PBS}$ of $\mathrm{pH}=7.4$ and 5.0 at $37^{\circ} \mathrm{C}$, respectively, irradiated with/without $1.5 \mathrm{~W} \mathrm{~cm}^{-2} \mathrm{NIR}$ for $5 \mathrm{~min}$ at the indicated time points.

chitosan chains in the nanogel drug carrier. Therefore, the DOX molecules have much higher mobility and thus can be released at a much faster rate at $\mathrm{pH}=5.0$ than at $\mathrm{pH}=7.4$ or 6.2. This $\mathrm{pH}$-responsive drug releasing behavior is very important for an intelligent drug carrier because the microenvironments of extracellular tissues of tumors and intracellular lysosomes and endosomes are acidic, which can potentially facilitate an active drug release from the PEG-chitosan@CDs hybrid nanogels..$^{[17,18]}$

Figure 7B compares the DOX releasing profiles from the PEG-chitosan@CDs hybrid nanogels dispersed in PBS solution ( $\mathrm{pH}=7.4$ and 5.0) at $37^{\circ} \mathrm{C}$ with and without use of NIR light irradiation for $5 \mathrm{~min}$ at certain time points, respectively. Without the use of NIR irradiation, the release of DOX from the hybrid nanogels is slow and reaches a nearly steady rate after $14 \mathrm{~h}$. In contrast, 5 min irradiation of NIR light can significantly speed up the release of DOX from the hybrid nanogels. When the NIR light was turned off, the drug release returns to its regular slow rate. Such a significantly enhanced drug releasing rate under 5 min exposures to NIR light should be attributed to the local heat produced by the efficient photothermal conversion of the fluorescent CDs embedded in the hybrid nanogels. Figure S8 in the Supporting Information confirms the highly efficient photothermal effect of the CDs exposed to NIR light at a power density of $1.5 \mathrm{~W} \mathrm{~cm}^{-2}$. In the presence of $50 \mathrm{mg} \mathrm{L}^{-1}$ of CDs, the temperature of water increases by $\approx 23{ }^{\circ} \mathrm{C}$ within
$5 \mathrm{~min}$. In contrast, the temperature change of pure water (control) was much less significant $\left(\approx 8^{\circ} \mathrm{C}\right)$ under the same irradiation conditions. The NIR light-induced local heat produced by the CDs immobilized in the nanogels not only weakens the drug-host interactions (e.g., hydrogen bonding and $\pi$ stacking, etc.) of DOX molecules with the CDs and PEG-chitosan network chains but also increases the mobility of DOX at elevated temperatures. In addition, the elevated temperatures also induce a shrinkage of the PEG-chitosan@CDs hybrid nanogels, which can sequester out the loaded DOX molecules. This NIR light triggerable drug releasing rate from the PEG-chitosan@ CDs hybrid nanogels offers additional fast-acting dosage under NIR photoactivation when necessary, thus can further improve the therapeutic efficacy when using the hybrid nanogels as a regular drug carrier for basal chemotherapy.

One advantage of the designed PEG-chitosan@CDs hybrid nanogels is their nontoxic materials. As shown in Figure 6, no signs of morphological damage to the cells were observed upon treatment with the PEG-chitosan@CDs hybrid nanogels, thereby demonstrating their noncytotoxicity. The in vitro cytotoxicity results shown in Figure 8A further confirm that the drug-free hybrid nanogels are nontoxic to the tested DU145 human prostate cancer cells. More than $98 \%$ of the DU145 human prostate cancer cells can survive upon $24 \mathrm{~h}$ treatment with the PEG-chitosan@CDs hybrid nanogels at concentrations
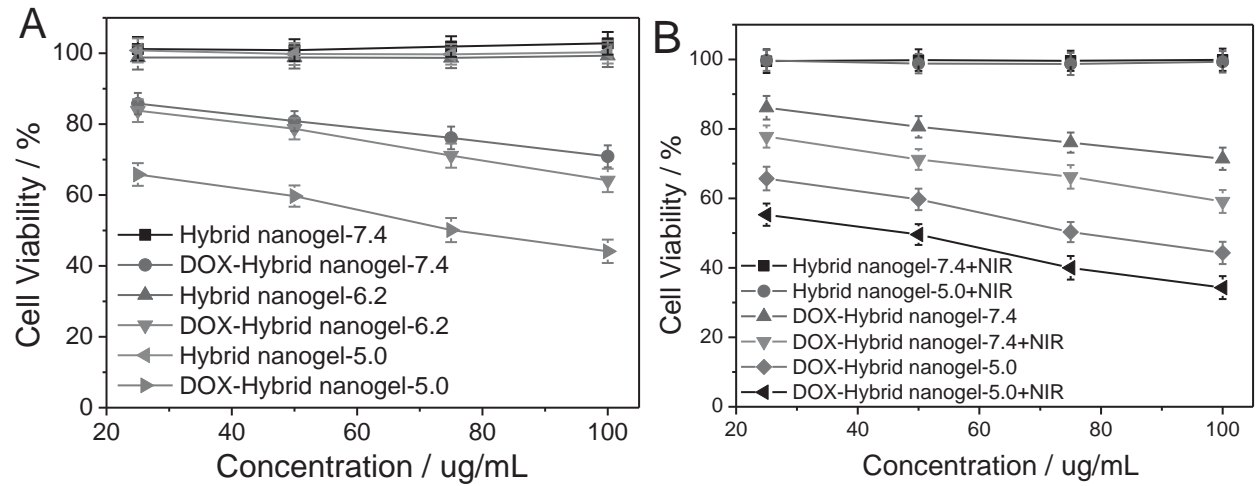

Figure 8. A) In vitro cytotoxicity of drug-free and DOX-loaded PEG-chitosan@CDs hybrid nanogels in cell culture media of different pH values; B) In vitro cytotoxicity of drug-free and DOX-loaded hybrid nanogels without and with the exposure to the $1.5 \mathrm{~W} \mathrm{~cm}{ }^{-2} \mathrm{NIR}$ light for 5 min, respectively. 

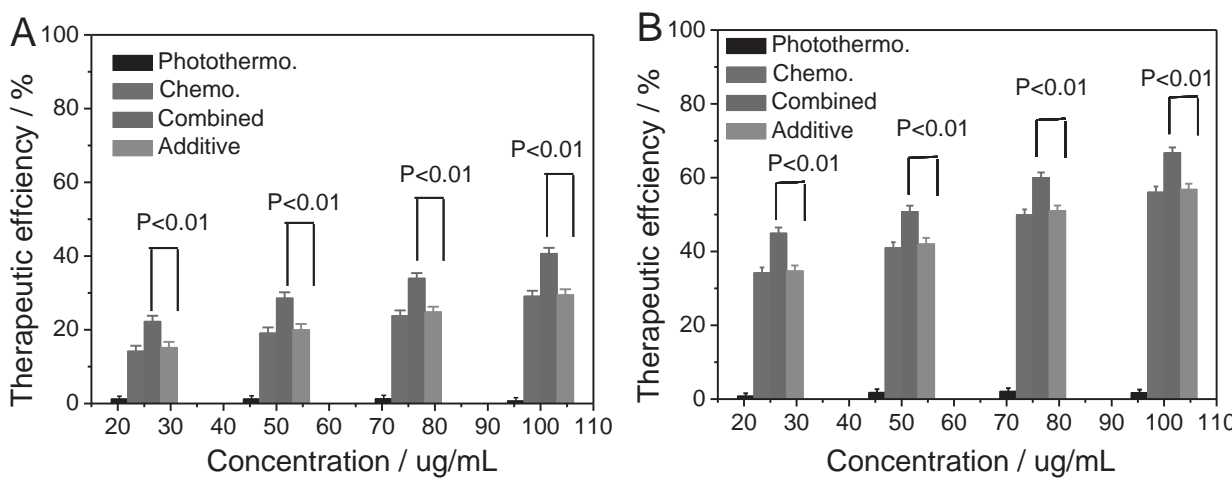

Figure 9. Therapeutic efficacies of PEG-chitosan@ @CDs hybrid nanogels as a drug carrier and photothermal therapy agent at A) pH=7.4 and B) pH=5.0, respectively, for chemo, photothermal, and their combined treatments. The additive therapeutic efficacies of independent chemo and photothermal treatments were estimated using the relation $T_{\text {additive }}=100-\left(f_{\text {chemo }} \times f_{\text {photothermal }}\right) \times 100$, where $f$ is the fraction of surviving cells after each treatment. The $t$-test was used to compare the therapeutic efficacies of combined treatment with the additive efficacies of chemo and photothermal treatments alone. All $p$-values are lower than 0.01 .

up to $100 \mu \mathrm{g} \mathrm{mL}-1$ even at $\mathrm{pH}=5.0$. In contrast, the cell viability drastically decreased when the cells were treated with the DOXloaded hybrid nanogels due to the drug effect of DOX molecules released from the hybrid nanogels. The $\mathrm{pH}$-dependent drug releasing rates were also demonstrated on the cell viability test of the DOX-loaded hybrid nanogels. When the $\mathrm{pH}$ was decreased from 6.2 to 5.0, the DOX-loaded hybrid nanogels can kill the cancer cells much more effectively because more DOX molecules can be released from the gel network at $\mathrm{pH}=5.0$ than at $\mathrm{pH}=6.2$ or 7.4. To investigate the photothermal effect of the hybrid nanogels, the DU145 cells were irradiated with $1.5 \mathrm{~W} \mathrm{~cm}^{-2}$ NIR light for $5 \mathrm{~min}$ at a certain time point during the incubation process with the drug-free and DOX-loaded hybrid nanogels, respectively. As shown in Figure 8B, the $5 \mathrm{~min}$ NIR irradiation alone only reduces a little bit on the viability of DU145 cells in normal culture medium when the hybrid nanogels carry no drug molecules. In contrast, the assistance of 5 min NIR irradiation on the DOX-loaded hybrid nanogels can significantly kill more tumor cells compared to the treatment without the use of 5 min NIR irradiation under the same conditions. The enhanced cytotoxicity of the DOX-loaded hybrid nanogels with the $5 \mathrm{~min}$ NIR irradiation should be attributed to the synergistic effect of photothermo/chemotherapy. Although the NIR light-induced local heat produced from the CDs embedded in the interior of nanogels had no much direct damage on cells, the local heat can significantly speed up the release of loaded DOX molecules to kill cancer cells. These results demonstrate that the PEG-chitosan@CDs hybrid nanogels as drug carriers can facilitate combined photothermal/ chemotherapy to provide high therapeutic efficacy.

Figure 9 shows a comparison of the therapeutic efficacies (calculated by subtracting the cell viability from 100\%) from the combined chemo-photothermal treatment with the additive therapeutic efficacies of independent chemo and photothermal treatments, which were estimated using the relation ${ }^{T}$ additive $=$ $100-\left(f_{\text {chemo }} \times f_{\text {photothermal }}\right) \times 100$, where $f$ is the fraction of surviving cells after each treatment. ${ }^{[6]]}$ The therapeutic efficacy of combined chemo-photothermal therapy with the DOX-loaded hybrid nanogels was significantly higher than the additive therapeutic efficacy of chemotherapy and photothermal therapy alone. When $t$-test is used to compare the efficacy of combined chemo-photothermal treatment with the additive efficacies of independent chemo and photothermal treatments, all $p$-values are lower than 0.01 , indicating a significant difference. Clearly, the PEG-chitosan@CDs hybrid nanogels as a drug carrier demonstrated a highly synergistic effect of the combined chemophotothermal treatment and thus achieved a high therapeutic efficacy, which will enable patients to lower down the dosage to reduce the systemic side effect.

In order to test the biocompatibility of the different samples in vivo, we performed histological analysis on various tissues (kidney and liver) of mice injected with the drug-free and DOX-loaded hybrid nanogels at a concentration of $0.1 \mathrm{mg} \mathrm{mL}^{-1}$, respectively, to identify any signs of acute toxicity. Tissues were harvested from mice $120 \mathrm{~h}$ after receiving the injection of hybrid nanogels, fixed in $10 \%$ formalin, embedded in paraffin, sectioned, and stained with hematoxylin and eosin (H\&E). Figure 10 shows the tissue sections from the injected and noninjected mice. The review results by a pathologist with expertise in veterinary pathology indicates that the tissues obtained from the mice injected with drug-free hybrid nanogels appear similar to those tissues from noninjected control animals, showing no evidence of toxicity. However, the kidney tissues from the mice injected with the DOX-loaded hybrid nanogels showed that the impaired glomeruli are filled with bright red granular proteinaceous material due to endothelial cell necrosis or sloughed pedicle, as well as necrosis of some hepatocytes, hydropic degeneration, and edema. The liver tissues obtained from the mice injected with DOX-loaded hybrid nanogels exhibit scattered bile canaliculi and damage in septal between lobes of liver. These results indicate that the drug-free PEG-chitosan@ CDs hybrid nanogels are nontoxic and have good biocompatibility. Meanwhile, these biocompatible hybrid nanogels can successfully carry drug molecules to tissues for chemotherapy.

\section{Conclusions}

In summary, highly stable, biocompatible, and multifunctional PEG-chitosan@CDs hybrid nanogels can be successfully 


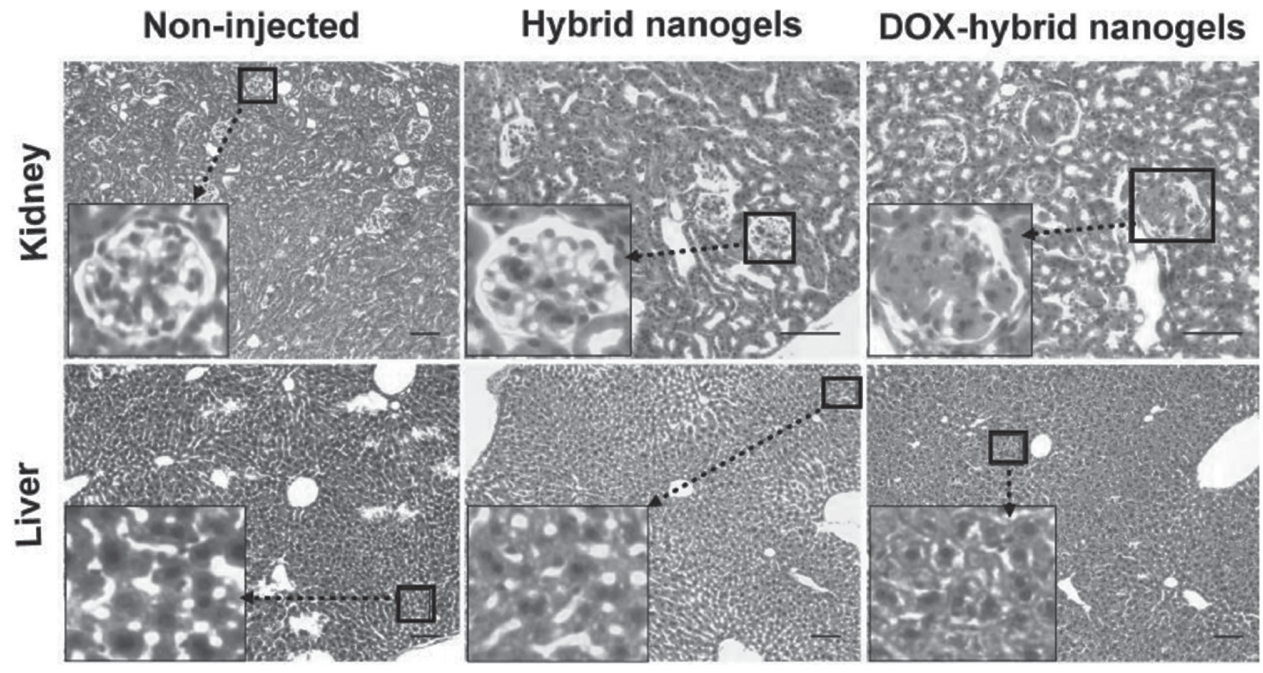

Figure 10. Representative H\&E stained tissue sections of mouse kidney and liver obtained from noninjected animals and from those injected with drug-free or DOX-loaded hybrid nanogels at a concentration of $0.1 \mathrm{mg} \mathrm{mL} \mathrm{L}^{-1}$. The scale bar $=50 \mu \mathrm{m}$. The inset at the lower left corner in each image was enlarged from the black line framed section indicated by arrow.

synthesized using a simple one-pot surfactant-free precipitation polymerization of PEG macromonomers complexed with chitosan chains and CDs. Combining the three functional building blocks, the PEG-chitosan@CDs not only maintain the properties from individual constituent but also display cooperative properties for simultaneous fluorescent $\mathrm{pH}$ sensing, TPF cellular imaging, and $\mathrm{pH} / \mathrm{NIR}$ light dual-responsive drug delivery. The embedded graphitic CDs with stable, excitation wavelength tunable, and upconverted PL properties not only provide the hybrid nanogels with high photothermal conversion ability and TPF cellular imaging ability but also enhance the loading capacity of the hybrid nanogels for hydrophobic anticancer drug molecules. The hybrid nanogels can enter the intracellular region and light up tumor cells under the excitations of both UV and NIR lasers. The $\mathrm{pH}$-sensitive volume phase transition of the hybrid nanogels can facilitate an on-site $\mathrm{pH}$-regulated drug release and modulate the surface properties of the embedded CDs to sense the environmental $\mathrm{pH}$ change over the physiologically important range of 5.0-7.4. Furthermore, the thermoresponsive properties of the hybrid nanogels can trigger the drug release under the irradiation of NIR light through the photothermal conversion ability of the embedded CDs. The hybrid nanogels enable a combined chemophotothermal treatment to provide a high therapeutic efficacy due to their highly synergistic effect. While the drug-free hybrid nanogels are nontoxic, the drug-loaded hybrid nanogels can deliver drug effectively to the liver and kidney tissues of mice. Such a multifunctional nanoplatform has great potential for in vivo medical diagnostics and therapy.

\section{Experimental Section}

Materials: $\mathrm{D}(\mathrm{t})$-Glucose was purchased from ACROS, and all other chemicals were purchased from Aldrich. 2-(2-methoxyethoxy) ethyl methacrylate $\left(\mathrm{MEO}_{2} \mathrm{MA}, 95 \%\right)$, oligo (ethylene glycol) methyl ether methacrylate $\left(\mathrm{MEO}_{5} \mathrm{MA}, M_{\mathrm{n}}=300 \mathrm{gmol}^{-1}\right.$ ), and poly(ethylene glycol) dimethacrylate (PEGDMA, $M_{\mathrm{n}} \approx 550 \mathrm{~g} \mathrm{~mol}^{-1}$ ) were purified with neutral
$\mathrm{Al}_{2} \mathrm{O}_{3}$. Chitosan was dissolved in acetic acid, then passed through a filter and dialyzed against distilled water for $3 \mathrm{~d}$ to remove free acetic acid. Doxorubicin hydrochloride, ammonium persulfate (APS), and $\mathrm{HC}$ (37\%) were used as received without further purification. The water used in all experiments was of Millipore Milli-Q grade.

Synthesis of Fluorescent CDs: Fluorescent CDs were synthesized via an acid-assisted ultrasonic and thermal treatment of glucose. In a typical synthesis, glucose $(2.70 \mathrm{~g})$ was dissolved in deionized water $(10 \mathrm{~mL})$. After intense sonication for $20 \mathrm{~min}, 30.0 \mathrm{~mL}$ of $\mathrm{HCl}(37 \mathrm{wt} \%)$ was slowly added into the above solution. The mixed solution was then treated ultrasonically for $8 \mathrm{~h}$ and transferred into a $50 \mathrm{~mL}$ Teflon-lined stainless autoclave. The precursor solution was heated to and maintained at $200{ }^{\circ} \mathrm{C}$. After $24 \mathrm{~h}$, the solution was cooled naturally to room temperature. The resulted CDs were purified with repeated centrifugation and redispersion in water for three cycles so that larger CDs could be removed from products. Finally, the aqueous dispersion of CDs was dialyzed for $7 \mathrm{~d}$ (Spectra/Pormolecularporous membrane tubing, cutoff $12000-14000)$ at room temperature $\left(\approx 22{ }^{\circ} \mathrm{C}\right)$. The dialytic aqueous dispersion of CDs was then collected and dried to get solid CDs.

Surfactant-Free Synthesis of PEG-Chitosan@CDs Hybrid Nanogels: Typically, CDs $(0.01 \mathrm{~g}), \mathrm{MEO}_{2} \mathrm{MA}\left(1.34 \times 10^{-3} \mathrm{~mol}\right), \mathrm{MEO}_{5} \mathrm{MA}$ $\left(6.60 \times 10^{-4} \mathrm{~mol}\right)$, PEGDMA $\left(6.00 \times 10^{-5} \mathrm{~mol}\right)$, and chitosan were dissolved in $198 \mathrm{~mL}$ deionized water $(\mathrm{pH} \approx 5.8)$ in a $250 \mathrm{~mL}$ roundbottom flask equipped with a stirrer, an $\mathrm{N}_{2}$ gas inlet, and a condenser. The mixture was heated to $70.0{ }^{\circ} \mathrm{C}$ under an $\mathrm{N}_{2}$ purge. After $1 \mathrm{~h}$, APS $(2 \mathrm{~mL}, 0.15 \mathrm{M})$ was added to the mixture to initiate the polymerization reaction. The reaction was allowed to proceed for $5 \mathrm{~h}$. The resultant reaction solution was centrifuged at $20000 \mathrm{rpm}\left(37^{\circ} \mathrm{C}, 30 \mathrm{~min}\right.$, Thermo Electron Co. SORVALLRC-6 PLUS superspeed centrifuge) with the supernatant discarded and the precipitate redispersed in $50 \mathrm{~mL}$ deionized water. After three repeated centrifugation/redispersion cycles, the resultant hybrid nanogel dispersion was diluted to a volume of $200 \mathrm{~mL}$ and then further purified to remove possible remained monomer and free CDs by $7 \mathrm{~d}$ of dialysis (Spectra/Por molecular porous membrane tubing, cutoff $12000-14000$ ) against very frequently changed water at room temperature $\left(22^{\circ} \mathrm{C}\right)$. The dialyzed water was monitored using UV-vis absorption spectrum. As shown in Figure S1 (Supporting Information), the characteristic absorption peak of CDs gradually disappeared after a continuous dialysis against the frequently changed water. The free CDs in the hybrid nanogel dispersions can be completely removed after about 3-4 d.

Drug Loading and Release of PEG-Chitosan@CDs Hybrid Nanogels: DOX was loaded into the hybrid nanogels by complexation method. 
The hybrid nanogel dispersion $(20 \mathrm{~mL})$ placed in a vial was stirred in a water bath $\left(10^{\circ} \mathrm{C}\right)$ for $30 \mathrm{~min}$. Then, DOX solution $(2.0 \mathrm{~mL}$, $1.0 \mathrm{mg} \mathrm{mL}^{-1}, \mathrm{pH}=7.4$ ) was added into the hybrid nanogel dispersion. After a continuous stirring for $24 \mathrm{~h}$, the DOX-loaded hybrid nanogel complexes were then separated from the dispersion solution by centrifugation $\left(37.0^{\circ} \mathrm{C}, 20000 \mathrm{rpm}\right.$, and $\left.30 \mathrm{~min}\right)$ and washed six times with distilled water to remove the unloaded DOX attached on the surface of hybrid nanogels. All the supernatant and washing solution was collected for the measurements of the drug loading content. The unloaded DOX presented in the supernatant was quantified by a UV-vis spectrophotometer at $480 \mathrm{~nm}$. The drug loading content of the hybrid nanogels was calculated by $\left(M_{0}-M_{t}\right) / M_{N} \times 100 \%$, where $M_{0}$ and $M_{t}$ are the total mass of DOX dissolved in the initial solution and remained in the supernatant solution, respectively. $M_{N}$ is the mass of the dried nanogels used in the loading process.

The in vitro NIR light/pH-responsive release of DOX from the hybrid nanogels were evaluated by the dialysis method. The purified DOX-loaded hybrid nanogels were redispersed in $25 \mathrm{~mL}$ PBS solution $(0.005 \mathrm{M}, \mathrm{pH}=7.4)$. First, two dialysis bags each filled with $5 \mathrm{~mL}$ diluted DOX-loaded hybrid nanogels were immersed in $50 \mathrm{~mL} 0.005 \mathrm{M}$ buffer solutions of $\mathrm{pH}=7.4$ at $37{ }^{\circ} \mathrm{C}$ with one of them exposed to an NIR lamp of an output power of $1.5 \mathrm{~W} \mathrm{~cm}^{-2}$ for $5 \mathrm{~min}$. Second, three dialysis bags each filled with $5 \mathrm{~mL}$ diluted DOX-loaded hybrid nanogels were immersed in $50 \mathrm{~mL} 0.005 \mathrm{M}$ buffer solutions with different $\mathrm{pH}$ values $(7.4,6.2$, and 5.0). The released DOX outside of the dialysis bag was sampled at defined time period and assayed by UV-vis spectrometry at $480 \mathrm{~nm}$. Cumulative release was expressed as the total percentage of drug released through the dialysis membrane over time.

Two-Photon Fluorescence (TPF) Cellular Imaging with PEG-Chitosan@ CDs Hybrid Nanogels as Labels: DU145 human prostate cancer cells were employed to evaluate the property of TPF imaging of the resultant PEG-chitosan@CDs hybrid nanogels. DU145 human prostate cancer cells were seeded into collagen-coated glass bottom dishes (MatTek) at a density of 4000 cells cm$~^{-2}$. The cells were grown for $24 \mathrm{~h}$ before incubated with the PEG-chitosan@CDs hybrid nanogel particles. Hybrid nanogels were suspended in cell culture media by extensive ultrasonication for $30 \mathrm{~min}$ and adjusted to a final concentration of $5 \mu \mathrm{g} \mathrm{mL}$. The cells were then incubated with the hybrid nanogels overnight before imaging. Live-cell TPF images were obtained using 20X (dry) or 60X (water immersion) objectives on a Leica SP8 2-Photon/ FLIM confocal microscope equipped with an environmental chamber to maintain the experimental environment at $37^{\circ} \mathrm{C}$. The excitation wavelength was $900 \mathrm{~nm}$ for TPF imaging.

In Vitro Cytotoxicity Test of the Drug-Free and DOX-Loaded Hybrid Nanogels with or without NIR Irradiation: In this study, DU145 human prostate cancer cells were cultured in the 96 wells microplate in $100 \mu \mathrm{L}$ medium containing about 2000 cells seeded into each well. After an overnight incubation for attachment, the medium was removed and another $100 \mu \mathrm{L}$ medium containing drug-free or DOX-loaded hybrid nanogels was added to make the final exact concentrations of

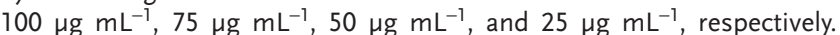
Wells that used the normal medium without any hybrid nanogels were used as control. For photothermal treatments, the cells were irradiated with $1.5 \mathrm{~W} \mathrm{~cm}^{-2}$ NIR light for $5 \mathrm{~min}$ in the middle of the incubation. After incubated for $24 \mathrm{~h}, 10 \mu \mathrm{L}$ of 3-(4,5-dimethyl-2-thiazolyl)-2,5diphenyltetrazolium bromide (MTT) solution (5 mg mL $\mathrm{mb}^{-1}$ in PBS) is added into the wells, which were continuously incubated in a humidified environment of $5 \% \mathrm{CO}_{2}$ and $37{ }^{\circ} \mathrm{C}$ for another $2 \mathrm{~h}$. The medium were then removed and $100 \mu \mathrm{L}$ of dimethyl sulfoxide solution is added. The plates were gently agitated until the formazan precipitate was dissolved, followed by measurement of optical density value by spectrophotometer at $570 \mathrm{~nm}$ and $690 \mathrm{~nm}$.

Histopathological Evaluation: After an intracardial perfusion of buffered 10\% formalin, whole organs (liver and kidney) of C57BL/ 6 mice were removed through necropsy and postfixed in the same fixative for $48 \mathrm{~h}$ and embedded in paraffin processed for histology, sliced into $5 \mu \mathrm{m}$ sections, and stained with hematoxylin and eosin (H\&E) according to standard clinical pathology protocols. A veterinary pathologist was then consulted to evaluate if any signs of acute toxicity were present in these clearance organs and cerebellum. Samples were submitted pathology assay $5 \mathrm{~d}$ after intravenous administration of drug-free hybrid nanogels $(n=3)$ and DOX-loaded hybrid nanogels $(n=3)$ with a concentration of $0.1 \mathrm{mg} \mathrm{mL}^{-1}$ and compared to both mice receiving no injection $(n=3)$.

Characterization: Transmission electron microscopy (TEM) images were taken on a FEI TECNAI transmission electron microscope at an accelerating voltage of $100 \mathrm{kV}$. High-resolution TEM images were characterized by JEM 2100 with an acceleration voltage of $200 \mathrm{kV}$. The UV-vis absorption spectra were obtained on a Thermo Electron Co. Helios $\beta$ UV-vis Spectrometer. The FTIR spectra were recorded with a Nicolet Instrument Co. MAGNA-IR 750 FTIR spectrometer. The UV light was provided by a $250 \mathrm{~W}$ high-pressure fluorescent $\mathrm{Hg}$ lamp. The PL spectra were obtained on a JOBIN YVON Co. FluoroMax-3 fluorospectrometer equipped with a Hamamatsu R928P photomultiplier tube, calibrated photodiode for excitation reference correction from 200 to $980 \mathrm{~nm}$, and an integration time of $1 \mathrm{~s}$. The photothermal experiments were conducted using a Philips infrared reflector lamp with a power density of $1.5 \mathrm{~W} \mathrm{~cm}^{-2}$ and a filter to block ultraviolet light. Dynamic light scattering (DLS) was performed on a standard laser light scattering spectrometer (BI-200SM) equipped with a BI-9000 AT digital time correlator (Brookhaven Instruments, Inc.) to measure the hydrodynamic diameter $\left(D_{h}\right)$ distributions. An He-Ne laser $(35 \mathrm{~mW}, 633 \mathrm{~nm})$ was used as the light source. The hybrid nanogels dispersion was passed through Millipore Millex-HV filters with a pore size of $0.45 \mu \mathrm{m}$ to remove dust before the DLS measurement. Confocal microscopic images were obtained using an Olympus Revolution XD Spinning Disk confocal microscope equipped with an environmental chamber to maintain the experimental environment at $37{ }^{\circ} \mathrm{C}$. Diode laser with a wavelength of $405 \mathrm{~nm}$ was used for the excitation.

\section{Supporting Information}

Supporting Information is available from the Wiley Online Library or from the author.

\section{Acknowledgements}

This research was supported by American Diabetes Association (Basic Science Award 1-12-BS-243) and PSC-CUNY Research Award. Principles of animal care and experimentation followed are those found in the Guide for the Care and Use of Laboratory Animals (DHEW Pub. No. (NIH) 85-23 revised 1996). College of Staten Island operates in accordance with an approved Animal Welfare Assurance on file with the Office for Protection from Research Risks. Animal Colony is under the supervision of veterinarians and the City University of New York Animal Care and Use Committee (ACUC), all of whom assure compliance with $\mathrm{NIH}$ and USDA guidelines.

Received: April 15, 2015

Revised: June 30, 2015

Published online: July 27, 2015

[1] A. Döring, W. Birnbaum, D. Kuckling, Chem. Soc. Rev. 2013, 42, 7391.

[2] M. Motornov, Y. Roiter, I. Tokarev, S. Minko, Prog. Polym. Sci. 2010, $35,174$.

[3] M. Oishi, Y. Nagasaki, Nanomedicine 2010, 5, 451.

[4] T. Sun, Y. S. Zhang, B. Pang, D. C. Hyun, M. Yang, Y. Xia, Angew. Chem., Int. Ed. 2014, 53, 12320.

[5] W. Wu, J. Shen, P. Banerjee, S. Zhou, Adv. Funct. Mater. 2011, 21, 2830 
[6] Y. Pan, Y. Chen, D. Wang, C. Wei, J. Guo, D. Lu, C. Chu, C. Wang, Biomaterials 2012, 33, 6570.

[7] S. C. Wei, M. Pan, K. Li, S. Wang, J. Zhang, C. Y. Su, Adv. Mater. 2014, 26, 2072

[8] H. Park, J. Yang, S. Seo, K. Kim, J. Suh, D. Kim, S. Haam, K. H. Yoo, Small 2008, 4, 192.

[9] A. Riedinger, M. P. Leal, S. R. Deka, C. George, I. R. Franchini, A. Falqui, R. Cingolani, T. Pellegrino, Nano Lett. 2011, 11, 3136.

[10] H. Kang, A. C. Trondoli, G. Zhu, Y. Chen, Y. J. Chang, H. Liu, Y. F. Huang, X. Zhang, W. Tan, ACS Nano 2011, 5, 5094.

[11] D. Peer, J. M. Karp, S. Hong, O. C. FaroKhzad, R. Margalit, R. Langer, Nat. Nanotechnol. 2007, 2, 751.

[12] E. Blanco, C. W. Kessinger, B. D. Sumer, J. Gao, Exp. Biol. Med. 2009, 234, 123.

[13] M. Stuart, W. Huck, J. Genzer, M. Müller, C. Ober, M. Stamm, G. Sukhorukov, I. Szleifer, V. Tsukruk, M. Urban, F. Winnik, S. Zauscher, I. Luzinov, S. Minko, Nat. Mater. 2010, 9, 101.

[14] M. A. Phillips, M. L. Gran, N. A. Peppas, Nano Today 2010, 5, 143.

[15] M. H. Xiong, Y. J. Li, Y. Bao, X. Z. Yang, B. Hu, J. Wang, Adv. Mater. 2012, 24, 6175

[16] W. Wu, N. Mitra, E. C. Y. Yan, S. Zhou, ACS Nano 2010, 4, 4831.

[17] D. Maciel, P. Figueira, S. Xiao, D. Hu, X. Shi, J. Rodrigues, H. Tomás, Y. Li, Biomacromolecules 2013, 14, 3140.

[18] C. Stefanadis, C. Chrysochoou, D. Markou, K. Petraki, D. B. Panagiotakos, C. Fasoulakis, A. Kyriakidis, C. Papadimitriou, P. K. Toutouzas, J. Clin. Oncol. 2001, 19, 676.

[19] I. F. Tannock, D. Rotin, Cancer Res. 1989, 49, 4373.

[20] C. Song, V. Appleyard, K. Murray, T. Frank, W. Sibbett, A. Cuschieri, A. Thompson, Int. J. Cancer 2007, 121, 1055.

[21] Y. T. Chang, P. Y. Liao, H. S. Sheu, Y. J. Tseng, F. Y. Cheng, C. S. Yeh, Adv. Mater. 2012, 24, 3309

[22] L. Qiu, T. Chen, I. Öçsoy, E. Yasun, C. Wu, G. Zhu, M. You, D. Han, J. Jiang, R. Yu, W. Tan, Nano. Lett. 2015, 15, 457.

[23] W. Li, P. Liao, C. Su, C. Yeh, J. Am. Chem. Soc. 2014, 136, 10062.

[24] Y. Wang, K. Wang, R. Zhang, X. Liu, X. Yan, J. Wang, E. Wagner, R. Huang, ACS Nano 2014, 8, 7870.

[25] Y. Wang, M. Shim, N. S. Levinson, H. Sung, Y. Xia, Adv. Funct. Mater. 2014, 24, 4206

[26] S. M. Lee, H. Park, K. H. Yoo, Adv. Mater. 2010, 22, 4049.

[27] F. F. Jöbsis vander-Vleit, J. Biomed. Opt. 1999, 4, 392.

[28] M. Ferrari, V. Quaresima, Neuroimage 2012, 63, 921.

[29] W. Wu, J. Shen, P. Banerjee, S. Zhou, Biomaterials 2010, 31, 7555.

[30] R. Guo, L. Zhang, H. Qian, R. Li, X. Jiang, B. Liu, Langmuir 2010, $26,5428$.

[31] W. Wu, J. Shen, P. Banerjee, S. Zhou, Biomaterials 2011, 32, 598.

[32] R. Chen, X. Zheng, H. Qian, X. Wang, J. Wang, X. Jiang, Biomater. Sci. 2013, 1, 285.

[33] J. You, P. Guo, D. T. Auguste, Angew. Chem. Int. Ed. 2013, 52, 4141.

[34] P. Shi, E. Ju, J. Ren, X. Qu, Adv. Funct. Mater. 2014, 24, 826.

[35] N. Khlebtsov, L. Dykman, Chem. Soc. Rev. 2011, 40, 1647.

[36] Y. Pan, S. Neuss, A. Leifert, M. Fischler, F. Wen, U. Simon, G. Schmid, W. Brandau, W. Jahnen-Dechent, Small 2007, 3, 1941.

[37] Y. P. Sun, B. Zhou, Y. Lin, W. Wang, K. A. S. Fernando, P. Pathak, M. J. Meziani, B. A. Harruff, X. Wang, H. Wang, P. G. Luo, H. Yang, M. Kose, B. Chen, L. Veca, S. Xie, J. Am. Chem. Soc. 2006, 128, 7756.
[38] S. Yang, X. Wang, H. Wang, F. Lu, P. G. Luo, L. Cao, M. J. Meziani, J. Liu, Y. Liu, M. Chen, Y. Huang, Y. Sun, J. Phys. Chem. C 2009, 113, 18110

[39] S. N. Baker, G. A. Baker, Angew. Chem., Int. Ed. 2010, 49, 6726.

[40] J. C. G. Esteves da Silva, H. M. R. Gonçalves, TrAC-Trends Anal. Chem. 2011, 30, 1327.

[41] S. K. Bhunia, A. Saha, A. R. Maity, S. C. Ray, N. R. Jana, Sci. Rep. 2013, 3, 1473

[42] L. Cao, M. J. Meziani, S. Sahu, Y. P. Sun, Acc. Chem. Res. 2013, 46, 171.

[43] L. Wu, M. Luderer, X. Yang, C. Swain, H. Zhang, K. Nelson, A. J. Stacy, B. Shen, G. M. Lanza, D. Pan, Theranostics 2013, 3, 677.

[44] P. G. Luo, F. Yang, S. T. Yang, S. K. Sonkar, L. Yang, J. J. Broglie, Y. Liu, Y. P. Sun, RSC Adv. 2014, 4, 10791.

[45] C. Ding, A. Zhu, Y. Tian, Acc. Chem. Res. 2014, 47, 20.

[46] Y. Huang, X. Zhou, R. Zhou, H. Zhang, K. Kang, M. Zhao, Y. Peng, Q. Wang, H. Zhang, W. Qiu, Chem. Eur. J. 2014, 20, 5640.

[47] H. Wang, F. Ke, A. Mararenko, Z. Wei, P. Banerjee, S. Zhou, Nanoscale 2014, 6, 7443.

[48] H. Nie, M. Li, Q. Li, S. Liang, Y. Tan, L. Sheng, W. Shi, S. Zhang, Chem. Mater. 2014, 26, 3104.

[49] H. Wang, J. Sheng, Y. Li, Z. Wei, G. Cao, Z. Gai, K. Hong P. Banerjee, S. Zhou, Biomater. Sci. 2014, 2, 915.

[50] B. Kong, A. Zhu, C. Ding, X. Zhao, B. Li, Y. Tian, Adv. Mater. 2012 24, 5844.

[51] J. Tang, B. Kong, H. Wu, M. Xu, Y. Wang, Y. Wang, D. Zhang, G. Zheng, Adv. Mater. 2013, 25, 6569.

[52] M. Zheng, S. Liu, J. Li, D. Qu, H. Zhao, X. Guan, X. Hu, Z. Xie, X. Jing, Z. Sun, Adv. Mater. 2014, 26, 3554.

[53] H. Wang, Y. Sun, J. Yi, J. Fu, J. Di, A. C. Alonso, S. Zhou, Biomaterials 2015, 53, 117

[54] M. Zeng, Z. P. Fang, J. Membr. Sci. 2004, 245, 95.

[55] L. Tian, D. Ghosh, W. Chen, S. Pradhan, X. Chang, S. Chen, Chem. Mater. 2009, 21, 2803.

[56] K. S. Novoselov, A. K. Geim, S. V. Morozov, D. Jiang, Y. Zhang, S. V. Dubonos, I. V. Grigorieva, A. A. Firsov, Science 2004, 306, 666.

[57] B. H. Tan, K. C. Tam, Adv. Colloid Interface Sci. 2008, 136, 25.

[58] J. F. Lutz, O. Akdemir, A. Hoth, J. Am. Chem. Soc. 2006, 128, 13046

[59] M. Shibayama, T. Tanaka, Adv. Polym. Sci. 1993, 109, 1.

[60] H. Wang, Z. Wei, H. Matsui, S. Zhou, J. Mater. Chem. A 2014, 2, 15740

[61] L. Cao, X. Wang, M. Meziani, F. Lu, H. Wang, P. Luo, Y. Lin B. A. Harruff, L. M. Veca, D. Murray, S. Xiu, Y. Sun, J. Am. Chem. Soc. 2007, 129, 11318

[62] X. Wang, L. Cao, S. Yang, F. Lu, M. Meziani, L. Tian, K. Sun, A. M. Bloodgood, Y. Sun, Angew. Chem. Int. Ed. 2010, 49, 5310.

[63] W. Jiang, B. Y. S. Kim, J. T. Rutka, W. C. W. Chan, Nat. Nanotechnol. 2008, 3, 145.

[64] A. N. Lukyanov, Z. Gao, L. Mazzola, V. P. Torchilin, Pharm. Res. 2002, 19, 1424

[65] X. Sun, Z. Liu, K. Welsher, J. Robinson, A. Goodwin, S. Zaric, H. Dai, Nano Res. 2008, 1, 203.

[66] G. M. Hahn, J. Braun, I. Har-kedar, Proc. Natl. Acad. Sci. USA 1975, 72, 937. 